\title{
探究 GNSS 技术在工程测绘中的特点及应用
}

\section{Exploring the Characteristics and Application of GNSS Technology in}

\section{Engineering Surveying and Mapping}

\author{
乔战伟
}

Zhanwei Qiao

河南省地质矿产勘查开发局第一地质矿 产调查院

中国・河南 洛阳 471000

The First Institute of Geology and Mineral Resources,Henan Bureau of Geology and Mineral Exploration and Development, Luoyang, Henan, 471000, China
【摘 要】和以往的测绘技能相比, GNSS 技术不管在准确效率层面还是在应用水平层面 都有着极大的优点和特征, 这也是其受到大规模使用的一个关键因素。论文将通过对其技 术的特点和使用规律的分析, 提出发展的策略,为其进一步发展指明方向。

【Abstract】Compared with previous surveying and mapping skills, GNSS technology has great advantages and characteristics in both accuracy and efficiency level and application level, which is also a key factor for its large-scale use. Through the analysis of its technical characteristics and usage rules, the paper puts forward the development strategy and points out the direction for its further development.

【关键词】GNSS 技术; 工程测绘; 特点; 应用

【Keywords \GNSS technology; engineering surveying and mapping; characteristics; application 【DOI】10.36012/se.v1i1.341

\section{GNSS 技术应用规律和特点}

\subsection{GNSS 技术应用规律}

GNSS 是指全球导航卫星系统，全球导航卫星系统定位 是利用一组卫星的伪距、星历、卫星发射时间等观测量,同时 还必须知道用户钟差。全球导航卫星系统是能在地球表面或 近地空间的任何地点为用户提供全天候的三维坐标和速度以 及时间信息的空基无线电导航定位系统 ${ }^{[1]}$ 。卫星导航定位技 术目前已基本取代了地基无线电导航、传统大地测量和天文 测量导航定位技术，并推动了大地测量与导航定位领域的全 新发展。当今, GNSS 系统不仅是国家安全和经济的基础设 施, 也是体现现代化大国地位和国家综合国力的重要标志。这 种技术的主要规律就是这个体系中的卫星发射出评估距离的 信号和导航资讯，卫星的方位资讯重点涵盖在导航电文中， 在此前提下合理通过距离交会测算法评估出站点 $\mathrm{P}$ 的实际 坐标。

\subsection{GNSS 技术的特点}

(1)GNSS 技术的定位精度系数十分突出。GNSS 系统重点 通过空间卫星、地面监控站及用户设备构建。用户设备在使用
时能够对接多个卫星, 摒除了部分系统化的差异[1]。这些自身 所具备的特点, 确保了该技术的高精度系数。正是工程测量工 作者清楚了解到该技术的特点, 进一步在实际工作中可以深 入发挥其优势, 这也正是该技术的特点所决定的。另外,由于 评估范畴的持续拓展, 该技术在数据评估中的精准度和准确 率会反映得更为明显。

(2) GNSS 技术能够精准让自动化操作得到实现。正是由 于在工程测绘中合理使用了这个技术，让即时智能化评估的 规划得到进一步落实 ${ }^{[3]}$ 。该技术在实际使用中十分便捷, 尤其 是由于中国持续开发以及增强的科学技术以及模式, 让该技 术的接收设备也更为小规模, 另外, 在实际操作中, 也更为简 便。而针对工程测绘工作者而言, 必须在现实的检测以及评估 时, 先把评估所必须的天线科学规划到对应的方位中, 这个时 候, 该技术既可以对观测中心的平面特点开展定位, 更关键的 是, 还可以给顾客带来立体的坐标导向。

(3)GNSS 技术具有检测效率高、时间不多的特征。在大部 分的工程检测中, 因为地质宽广, 以往的测绘技能无法对所有 地区开展合理评估, 需要对施工场地切割为几个领域开展合 理的地质检测, 最后把评估指标总结起来, 工作十分烦琐。不 
仅要有精准的系数, 同时, 还必须具备检测时间少和评估效率 高的特征。

(4)GNSS 技术能够让 $24 \mathrm{~h}$ 工作得到实现, 只要观测环境符 合标准, 观测就能够不断开展, 不会受到白天和黑夜的影响, 也不会受到任何恶劣天气的影响。在以往评估中发现, 因为地 势的繁杂、地面障碍的阻碍而导致的影响十分严重, 往往一出 现这种情况就无法正常工作。而在 GNSS 技术中, 只要让其基 本工作环境得到满足, 其也可以开展精准定位职能, 顺利开展 工作。这个特征极大降低了检测工作的设置时间。

(5)作用范畴大。城市间的用地高速朝郊外偏远地区发展, 建筑工地地形图检测需要提升; 大范围的施工地点必须设计 施工控制系统, 以往的主要控制系统经常无法让这些需要得到 满足。通过 GNSS 技术可以高效处理这些状况, 其具备别的测 量手段不能超越的优点, 不必和以往测量那般进行过渡点的设 计以及需要测量点之间的彼此通视水平, 同时经济投入不高。

\section{2 工程测量中 GNSS 技术的应用}

\section{1 选点并做好标志}

GNSS 测量中心具备十分灵敏的网络框架系统, 同时其 内部无法开展彼此之间的通视, 因此, 必须进行选点工作, 给 深人开展评估打下坚定的基础。和一般的测量技能比起来, 该 技术降低了视标建立开支, 也就是让工作的高效率得到实现, 同时还降低了经济投入。同时也发现, 该技术在工作过程中具 备自己独特的标准。

\subsection{GNSS 技术进行施工放样}

建筑物施工放样是和施工方案、步骤相协调的, 涵盖主要 施工放样、上面框架以及高距离建筑施工放样。在刚开始进行 测量时, 建筑物施工放样涵盖平面方位和孔装的放样, 工作内 容涵盖放样基槽(基坑)开挖边线、控制开挖深度、放样基层施 工高程和放样基础模板位置等。在地形波动大的场地, 直接通 过该技术放样具备极大的优点, 以往的全站设备往往必须把 设计位置放样出来, 往往必须让目标来回移动, 同时工作时必 须通过 2 3 人进行, 另外, 在开展放样的时候, 必须保障所有点 之间具备和谐的通视成效,然而这种手段的生产水平不高,

\section{3 对测量数据进行科学合理的处理}

必须对所有指标的齐全化、精准度和稳定性开展评估。必 须在评估地点当中迅速、详细评估作业记录指标。针对部分重 点场所, 还能够通过实际工作开展重新评估。必须严谨根据有 关技能要求来评估所有职能是否符合规范, 来最大水平降低 评估中的误差。接着, 必须通过平差统计手段对指标开展合理 处理。该技术所使用的测量手段, 是在评估时间实现 $15 \mathrm{~s}$ 的时
候, 系统会智能化开展工作, 在实际工作时, 利用电脑会智能 化对指标开展完善以及处理, 其自动化以及高效化也是其可 以大规模使用在工程测绘行业的关键要素之一。针对精度的 实际标准和软件的作用, 根据精度标准和实际问题, 对下载指 标开展调研, 待测系数、收玫系数和属性都和有关标准相融 合,才可以开展接下来的输送工作。

\section{4 加强专业人才的培养}

首先, 要进一步鼓励优秀测绘人员积极学习 GNSS 测绘 技术, 通过单位之间交流学习或者组织深造的形式, 针对性地 培养优秀人才。其次, 让优秀的 GNSS 测绘人员对道路测量员 工进行培训, 让广大测量人员加入到 GNSS 测绘技术的学习 行列, 并由优秀 GNSS 测绘人员作为技术指导, 通过实践提高 全体测绘人员的 GNSS 测绘技术。

\section{5 加大技术创新的研究}

由于社会经济的持续推进, 科技资讯技能的开发也发生 极大的转化。该技术即使已经逐渐完善并使用到工程测绘中, 但是还具备相对的优化空间。中国道路测绘部门, 必须在中国 工作中最大水平尽心对该技术开展进一步分析, 主动试着对该 技术进行创新, 让该技术可以再发展出一个全新水平。

\section{6 加强宣传力度}

提高 GNSS 测绘技术的推广, 既可以引进更多综合人才 来到这个领域, 同时可以让大家了解, 中国测绘工作发展的稳 固性以及创新理念。工程测绘工作的发展, 需要让整个社会知 晓, 在推广工程测绘时, 建设机构可以通过该技术的推广并进 行进一步宣传。

\section{3 结语}

和以往的测绘技能比起来, GNSS 技术不管在准确效率层 面还是在应用水平层面都有着极大的优点和特征, 这也是其 受到大规模使用的一个关键因素, 想要进一步确保其使用水 平, 落实对应的手段十分关键。由于科学技术的持续拓展, 所 有技术都获得了持续优化, 该技术的使用成效在今后的发展 中也会获得很大水平的提升, 这为中国工程测绘行业的开发 提供了更加充分的条件。

\section{参考文献}

[1]黄小梅.刍议 GNSS 技术在工程测绘中的应用与改进 [J].江西 建材,2015(2):209.

[2]肖称生. 地理信息技术在工程测绘中的应用[J].江西建材, 2015 (4):206-207.

[3]查立. 浅谈 GNSS 测量技术在工程测绘中的应用[J]. 科学中国 人,2015(32):14-15. 